\title{
Specificity of Glomerular Targeting by Olfactory Sensory Axons
}

\author{
Helen B. Treloar, ${ }^{1}$ Paul Feinstein, ${ }^{2}$ Peter Mombaerts, ${ }^{2}$ and Charles A. Greer ${ }^{1}$ \\ ${ }^{1}$ Department of Neurosurgery and Section of Neurobiology, Yale University School of Medicine, New Haven, Connecticut \\ 06520-8082, and ${ }^{2}$ The Rockefeller University, New York, New York 10021
}

\begin{abstract}
Axons from olfactory sensory neurons (OSNs) expressing a specific odorant receptor (OR) project to specific subsets of glomeruli in the olfactory bulb (for review, see Mombaerts, 1999, 2001). The aim of this study was to examine the trajectories that subsets of axons from OSNs expressing the same OR follow within the olfactory nerve and olfactory nerve layer (ONL) of adult mice. Using confocal microscopy, we generated serial reconstructions of axons from M72-IRES-tauGFPexpressing OSNs as they coursed within the ONL and into glomeruli. GFP-expressing axons were loosely aggregated in the outer ONL; however, as they entered the inner ONL, the majority fasciculated with other GFP-expressing axons before entering the glomerular neuropil. Although the vast majority of axons entered the glomerulus from the directly apposed ONL, some followed tortuous courses through and/or around adja-
\end{abstract}

Olfactory sensory neurons (OSNs) detect odors by way of seventransmembrane G-protein-coupled odorant receptors (ORs) located in the cilia emanating from their dendritic knob (Firestein, 2001). The OSN axons project to the olfactory bulb (OB) in which they synapse with the dendrites of mitral-tufted cells in spherical bundles of neuropil, the glomeruli. Converging lines of evidence suggest that glomeruli are basic functional units in the $\mathrm{OB}$ and that patterns of glomerular activity underlie odor coding (Leveteau and MacLeod, 1966; Shepherd, 1981; Guthrie et al., 1993; Rubin and Katz, 1999; Uchida et al. 2000).

A seminal advance in understanding glomerular organization was the discovery that axons from OSNs that express the same OR gene generally converge onto two or a few specific glomeruli in the OB (Ressler et al., 1994; Vassar et al., 1994; Mombaerts et al., 1996). These axons can be imaged and the innervation of the glomeruli studied by placing a reporter, taulacZ or tauGFP, under the control of a specific OR gene (Mombaerts et al., 1996; Potter et al., 2001). Recently, there have been reports of additional smaller secondary glomeruli that appear to be innervated by labeled OSN axons (Royal and Key, 1999; Strotmann et al., 2000; Pyrski et al., 2001; Schaefer et al., 2001).

The family of OR genes constitutes unique molecular markers that also influence the projection of OSN axons to specific glomeruli. Mombaerts et al. (1996) and Wang et al. (1998) showed

Received Nov. 13, 2001; revised Jan. 14, 2002; accepted Jan. 15, 2002.

This work was support in part by National Institutes of Health Grants DC00210 (C.A.G.), DC03452 and DC03596 (P.M.), and DC03887 (C.A.G., P.M.) and a Brown-Coxe Fellowship to H.B.T. We thank Christine Kaliszewski and Kara Salvagno for the electron microscopy, Dolores Montoya for technical help, and Janice Mitchell for administrative support.

Correspondence should be addressed to Dr. Charles A. Greer, Department of Neurosurgery, Yale University School of Medicine, P.O. Box 208082, 333 Cedar Street, New Haven, CT 06520-8082. E-mail: charles.greer@yale.edu.

Copyright (C) 2002 Society for Neuroscience $0270-6474 / 02 / 222469-09 \$ 15.00 / 0$ cent glomeruli before terminating in the target glomerulus. Similar observations were made on subpopulations of axons in M71-IRES-tauGFP and P2-IRES-tauGFP mice. Ultrastructural analyses of labeled M72 glomeruli showed no evidence of axodendritic synapses other than those with GFP-labeled axon terminals. These data are consistent with the notion that OSN axons are highly precise in targeting glomeruli and that glomeruli, in turn, are highly homogeneous with regard to the OR expressed by the innervating OSNs. Because some single axons could follow idiosyncratic trajectories to the target glomerulus, it appears that stable homotypic fasciculation is not a prerequisite for correct targeting.

Key words: axon targeting; GFP; electron microscopy; odor receptors; olfaction; synapse that genetic replacements of OR coding regions cause axons to target different glomeruli and that, when the OR coding region is deleted, axons distribute broadly and fail to converge to a glomerulus. Although the full scope of mechanisms that may be used by the ORs to influence glomerular targeting is not known, increasing evidence does suggest that functional activity may be important (Zheng et al., 2000; Zhao and Reed, 2001).

The aim of this study is to examine, at high resolution, the projections of specific populations of OSN axons to the OB. Although light microscopy indicates that all of the axons from OSNs that express the same OR converge onto a few glomeruli, the converse is not known: do all of the axons converging onto a glomerulus originate from OSNs that express the same OR? Gene-targeted mice in which the green fluorescent protein (tauGFP) is expressed along with a specific OR were examined using the following: (1) intrinsic GFP fluorescence and immunocytochemistry with confocal microscopy and (2) ultrastructural immunolocalization of GFP to examine the projection patterns and synaptic organization of OSNs expressing the reporter. Analysis of the trajectories of GFP-expressing axons in the olfactory nerve layer (ONL) and glomerular layer (GL) reveal that all GFP-expressing axons target the appropriate glomeruli. However, stable homotypic fasciculation between axons from OSNs expressing the same OR does not appear to be a prerequisite for correct targeting. Single axons often followed tortuous or isolated trajectories before entering the appropriate glomerulus. Analysis of the ultrastructural immunolocalization of GFP within M72 glomeruli suggest that the majority, if not all, of the OSN axons innervating a glomerulus express the same OR. Together, these data argue strongly that the primary olfactory projection is highly specific and that stable homotypic fasciculation, per se, is not a prerequisite for correct targeting. 


\section{MATERIALS AND METHODS}

\section{Animals}

Adult (postnatal days 30-90) M72-IRES-tauGFP $(n=9)$, M71-IRES$\operatorname{tauGFP}(n=3)$, and P2-IRES-tauGFP $(n=3)$ mice in a mixed $129 / \mathrm{Sv} \times$ C57BL/6 background were anesthetized with sodium pentobarbital (80 $\mathrm{mg} / \mathrm{kg}$, i.p.; Nembutal; Abbott Laboratories, Chicago, IL) and perfused using a low-/high-pH paraformaldehyde (PFA) fixation strategy (adapted from Berod et al., 1981). This strategy was adopted after preliminary studies indicated that ultrastructural immunolocalization of GFP was compromised in tissue perfused with fixatives containing glutaraldehyde. Tissue was initially perfused with $4 \%$ PFA in PBS $(0.1 \mathrm{M}$ phosphate buffer and $0.9 \% \mathrm{NaCl}_{2}$ ) at $\mathrm{pH} 6.5$ for $5 \mathrm{~min}$, followed by $4 \%$ PFA in PBS at $\mathrm{pH}$ 10.5. Brains were removed and post-fixed overnight at $4^{\circ} \mathrm{C}$ in the second perfusate. All tissue was rinsed for a minimum of $2 \mathrm{hr}$ in PBS after fixation before processing for microscopy. All procedures undertaken in this study were approved by the Animal Care and Use Committees of both the Yale University and The Rockefeller University and conformed to National Institutes of Health guidelines.

\section{Sectioning}

OBs of M72-IRES-tauGFP mice that were to be processed for immunoelectron microscopy $(n=3)$, as well as M72-IRES-tauGFP, M71-IREStauGFP, and P2-IRES-tauGFP OBs for imaging of GFP using the confocal microscope ( $n=3$ for each group), were embedded in $2 \%$ agarose in PBS and serially sectioned in the coronal plane $(50 \mu \mathrm{m}$ sections) using a Pelco (Redding, CA) 101 Vibratome. M72-IREStauGFP OBs $(n=3)$ that were to be immunostained and imaged on the confocal microscope were cryoprotected by immersion in $30 \%$ sucrose in PBS at $4^{\circ} \mathrm{C}$ until tissue sank. Tissue was embedded in OCT compound (Sakura Finetek, Torrance, CA) and frozen in a slurry of $100 \%$ ethanol and dry ice. Tissue was serially sectioned in the coronal plane $(20 \mu \mathrm{m}$ sections) using a Reichert-Jung 2800 Frigocut E cryostat. Section were thaw mounted onto microscope slides coated with $2 \%$ gelatin and $0.1 \%$ chromalum, air dried, and stored at $-20^{\circ} \mathrm{C}$ until needed.

\section{Imaging of M72-IRES-tauGFP glomeruli}

Intrinsic GFP fluorescence was imaged in serial coronal vibratome sections using a Bio-Rad (Hercules, CA) confocal microscope. Briefly, vibratome sections through M72, M71, and P2 glomeruli were mounted in $40 \%$ glycerol between two coverslips, and confocal images were collected at $2 \mu \mathrm{m}$ intervals through the entire glomerular volume (typically contained within three $50 \mu \mathrm{m}$ vibratome sections). Axons coursing in the ONL were imaged in the same way.

\section{Immunocytochemistry}

Confocal microscopy. Serial $20 \mu \mathrm{m}$ sections of M72 glomeruli were immunostained with polyclonal olfactory marker protein (OMP) antibodies (generous gift from Dr. Frank Margolis, University of Maryland at Baltimore, Baltimore, MD) to identify mature OSN axons (Keller and Margolis, 1975). Briefly, tissue was thawed and air dried and then incubated with $2 \%$ bovine serum albumin (BSA) (Sigma, St. Louis, MO) in TBST $[0.1 \mathrm{M}$ Tris buffer and $0.9 \%$ saline, $\mathrm{pH} 7.4$ (TBS), with $0.3 \%$ Triton X-100 (Sigma)] for 30 min to block nonspecific binding sites. The tissue was incubated in anti-OMP (1:800) for $2 \mathrm{hr}$ at room temperature. Sections were then washed three times in TBST for $5 \mathrm{~min}$. They were incubated in rabbit anti-goat $\mathrm{IgG}$ antibodies conjugated to Alexa-568 (Molecular Probes, Eugene, OR) diluted 1:200 in blocking buffer for $1 \mathrm{hr}$ at room temperature. Sections were washed (as above), rinsed in TBS, mounted in Vectashield mounting media (Vector Laboratories, Burlingame, CA), and coverslipped, and the edges of the coverslips were sealed with liquid nail enamel. Stained sections were analyzed using a Bio-Rad MRC-600 laser scanning confocal microscope. Digital images were collected from a single optical plane, $\sim 1 \mu \mathrm{m}$ thick.

Electron microscopy. Coronal vibratome sections of the OB were freefloating immunostained with polyclonal anti-GFP antibodies. Briefly, tissue was incubated with 2\% BSA (Sigma) in PBS for 30 min to block nonspecific binding sites. The tissue was incubated in a rabbit polyclonal anti-GFP antibody (Molecular Probes), diluted 1:1000 in blocking buffer for $2 \mathrm{hr}$ at room temperature. Sections were then washed three times in PBS for $5 \mathrm{~min}$. They were incubated in biotin-conjugated goat anti-rabbit IgG secondary antibodies (1:100; Vector Laboratories) for $1 \mathrm{hr}$ at room temperature. Sections were washed (as above) and incubated with ABC reagent (prepared by diluting both solution $A$ and solution $B$ at 1:50 in blocking buffer; Vector Laboratories) for $1 \mathrm{hr}$ at room temperature.
Sections were washed again (as above) and given a final rinse in PBS. Peroxidase activity was visualized by incubating tissue in $0.05 \% 3.3^{\prime}$ diaminobenzidine tetrahydrochloride and $0.005 \% \mathrm{H}_{2} \mathrm{O}_{2}$ in TBS. Reaction was stopped in PBS and then was incubated for $1 \mathrm{hr}$ in $2 \%$ glutaraldehyde in PBS. Sections were washed again (as above) and immediately processed for electron microscopy.

\section{Electron microscopy}

Immunostained sections were processed for electron microscopy as described previously (Montague and Greer, 1999). Briefly, stained tissue was post-fixed with osmium tetroxide, dehydrated through graded alcohols, and polymerized in Epon between glass slides and coverslips coated previously with Liquid Release Agent (Electron Microscopy Sciences, Fort Washington, PA). Smaller regions containing stained axons in the olfactory nerve and glomerular layers were removed and reembedded on blank Epon blocks for thin sectioning and conventional electron microcopy. Thin sections $(0.07 \mu \mathrm{m})$ were examined on a Jeol (Peabody, MA) 1200 electron microscope.

\section{Image preparation}

Using Confocal Assistant 4.02 software (Bio-Rad), serial optical sections (Z-series) obtained with the confocal microscope were projected into single images, and double-label confocal images were pseudocolored and merged. Electron micrograph negatives were scanned using a UMAX flat bed scanner. All digital images were color balanced using Adobe Photoshop 5.0 (Adobe Systems, San Jose, CA). The composition of the images was not altered in any way. Plates were constructed using Corel Draw 8.0 (Corel, Ottawa, Ontario, Canada).

\section{RESULTS \\ Axon trajectories}

To examine the trajectories of axons of neurons expressing the M72 OR, we used gene-targeted mice that express GFP from a bicistronic message produced under the control of the M72 OR promoter (Potter et al., 2001). Intrinsic GFP fluorescence was imaged with a confocal microscope. Serial $50 \mu \mathrm{m}$ vibratome sections of the OBs of these mice were collected and mounted between two coverslips. The axons were tracked through the ONL to their terminations in glomeruli. Within a section, axons were imaged at $2 \mu \mathrm{m}$ intervals throughout the depth of the tissue. These Z-series of images were projected into a single image (Fig. $1 A$ ). Within the ONL, the majority of axons were observed to project individually, or in very small fascicles, in the outer ONL (arrows), but, as they entered the inner ONL, they fasciculated (white arrowheads). To distinguish the trajectory of individual axons, we examined axons in the ONL (Fig. $1 A$, box) in single optical scans $(\sim 1.0 \mu \mathrm{m}$ thick) from Z-series (Fig. $1 B-I)$. Following the axons through serial optical sections from rostral to caudal (i.e., from $B$ to $I$ ), it can be seen that they course individually in the outer ONL (arrows). However, as the axons begin to enter the inner ONL, they form small fascicles (Fig. $1 B-E$, white arrowheads) that merge into larger fascicles (Fig. $1 F-I$, white arrowheads) before terminating in the glomerulus.

To further characterize the trajectory of axons expressing GFP in these mice, immunocytochemistry was used together with electron microscopy. At the ultrastructural level, axons expressing GFP demonstrated peroxidase staining along the microtubules (Fig. 2A). Because tau is targeted to microtubules, the tauGFP fusion protein is expected to be highly localized on microtubules. In the outer ONL, isolated axons expressing GFP (Fig. $2 A$, white asterisk) were observed coursing within bundles of unstained OSN axons (e.g., black asterisks) and could be followed for long distances in the ONL. However, within the inner ONL (Fig. 2B), stained axons (e.g., white asterisks) were generally found together in fascicles, although unstained axons (black asterisks) were present as well. 

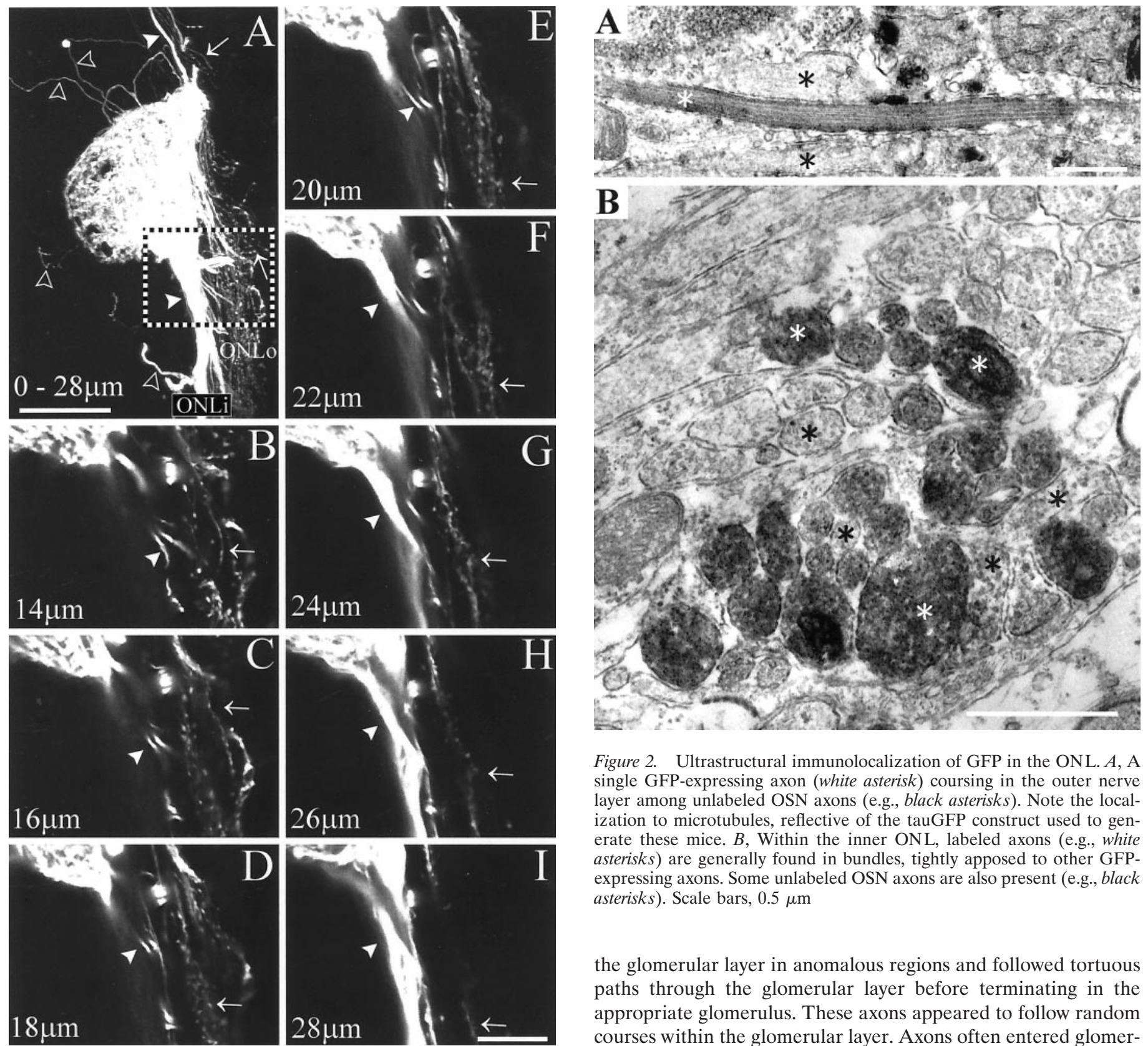

Figure 2. Ultrastructural immunolocalization of GFP in the ONL. A, A single GFP-expressing axon (white asterisk) coursing in the outer nerve layer among unlabeled OSN axons (e.g., black asterisks). Note the localization to microtubules, reflective of the tauGFP construct used to generate these mice. $B$, Within the inner ONL, labeled axons (e.g., white asterisks) are generally found in bundles, tightly apposed to other GFPexpressing axons. Some unlabeled OSN axons are also present (e.g., black asterisks). Scale bars, $0.5 \mu \mathrm{m}$

the glomerular layer in anomalous regions and followed tortuous paths through the glomerular layer before terminating in the appropriate glomerulus. These axons appeared to follow random courses within the glomerular layer. Axons often entered glomeruli from within the glomerular layer at multiple entry sites (Fig. $1 A$, open arrowheads).

To determine whether all of these atypically projecting axons

Figne 1. Trajectories of M72-IRES-tauGFP axons in the ONL of the adult $\mathrm{OB}$. $A$, Fifteen serial $2 \mu \mathrm{m}$ optical sections through part of a medial M72-IRES-tauGFP glomerulus that have been projected into a single image. Axons can been seen coursing individually in the outer ONL (ONLo; arrow) but appear to be mostly fasciculated in the inner ONL (ONLi; white arrowheads). Some axons follow torturous paths to the glomerulus through the glomerular layer (open arrowheads). B-I, To resolve individual fibers, we examined confocal images of single optical planes from the projected stack (boxed region in $A$ ). Individual fibers can be seen in the outer ONL (arrows; $B-I$ ), whereas the majority of axons fasciculate after entering the inner ONL (white arrowheads; $B-I$ ). Note that, in all panels, the outer ONL lies to the right of the inner ONL. Scale bars: $A, 50 \mu \mathrm{m} ; B-I, 20 \mu \mathrm{m}$.

\section{Glomerular targeting}

Although the majority of axons projected to glomeruli along this stereotypical trajectory, a small subset of axons was identified that did not (Figs. 1A, open arrowheads, 3, white arrowheads). In all glomeruli examined $(n=12)$, axons were observed that entered terminated within the M72 glomerulus, serial $50 \mu \mathrm{m}$ vibratome sections through M72-IRES-tauGFP glomeruli were imaged at 2 $\mu \mathrm{m}$ optical intervals, and GFP-expressing axons were followed through successive sections (Fig. 3). All of the GFP-expressing axons appear to innervate the M72 glomerulus (Fig. $3 a-a^{\prime \prime}, b-b^{\prime \prime}$, $c-c^{\prime \prime}, D-D^{\prime \prime}, d-d^{\prime \prime}$, arrowheads). Although it is impossible to assign polarity to axons that express GFP, it seems likely that axons are entering rather than exiting glomeruli as they can be traced from the ONL and the glomeruli. It is particularly evident in Figure 3 that the morphologies of both medial $\left(A^{\prime}, B^{\prime}\right)$ and lateral $\left(C^{\prime}, D^{\prime}\right)$ M72 glomeruli vary in both size and shape.

To determine whether atypical axonal projections are unique to axons from M72-expressing OSNs or whether these types of projections were the rule, we examined GFP expression in both M71-IRES-tauGFP mice (a highly related OR that is expressed in 


\section{Medial}
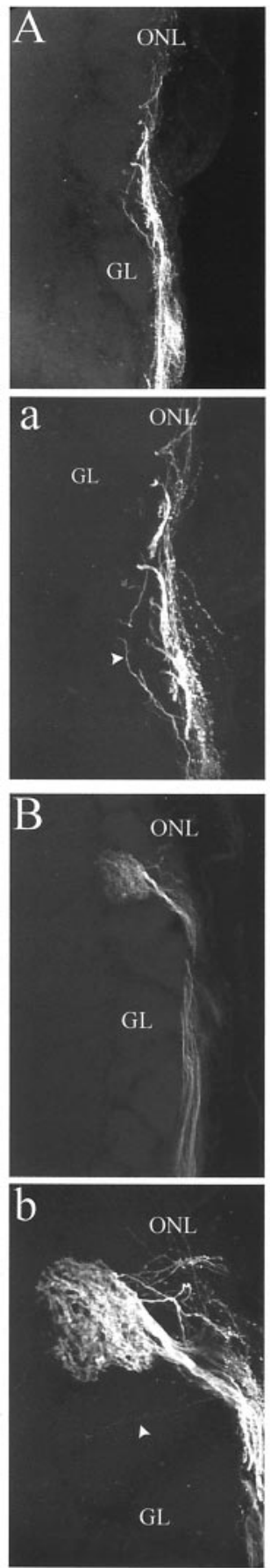
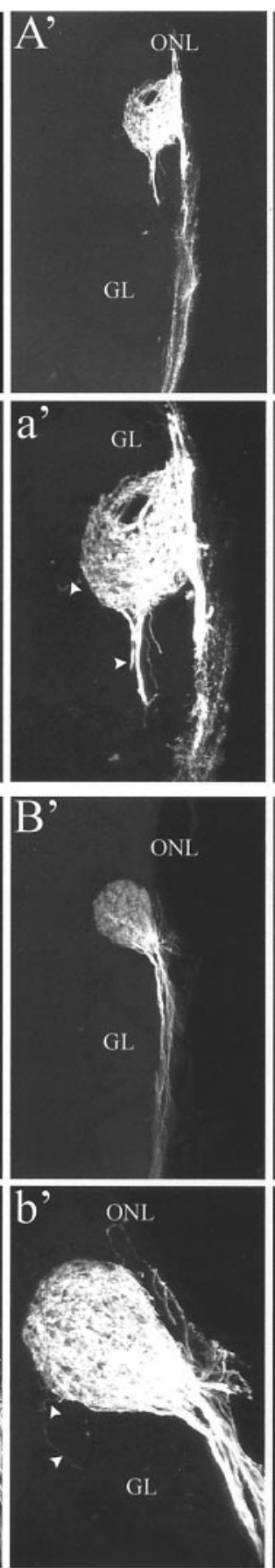
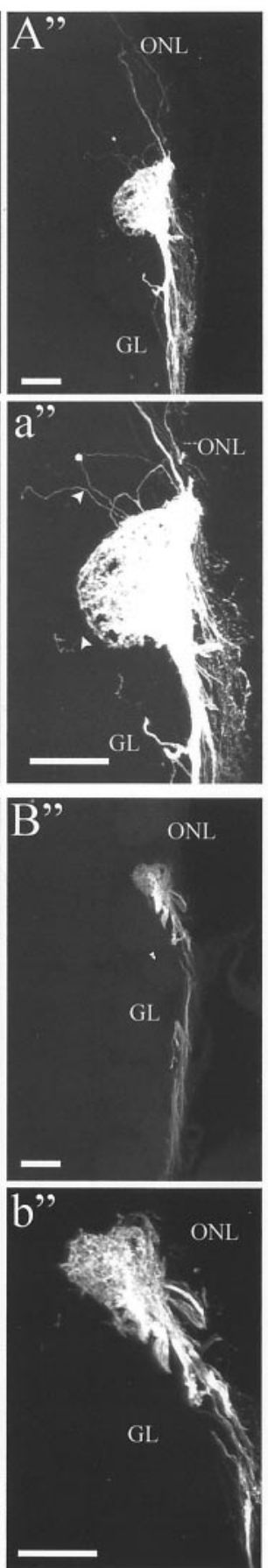

Lateral
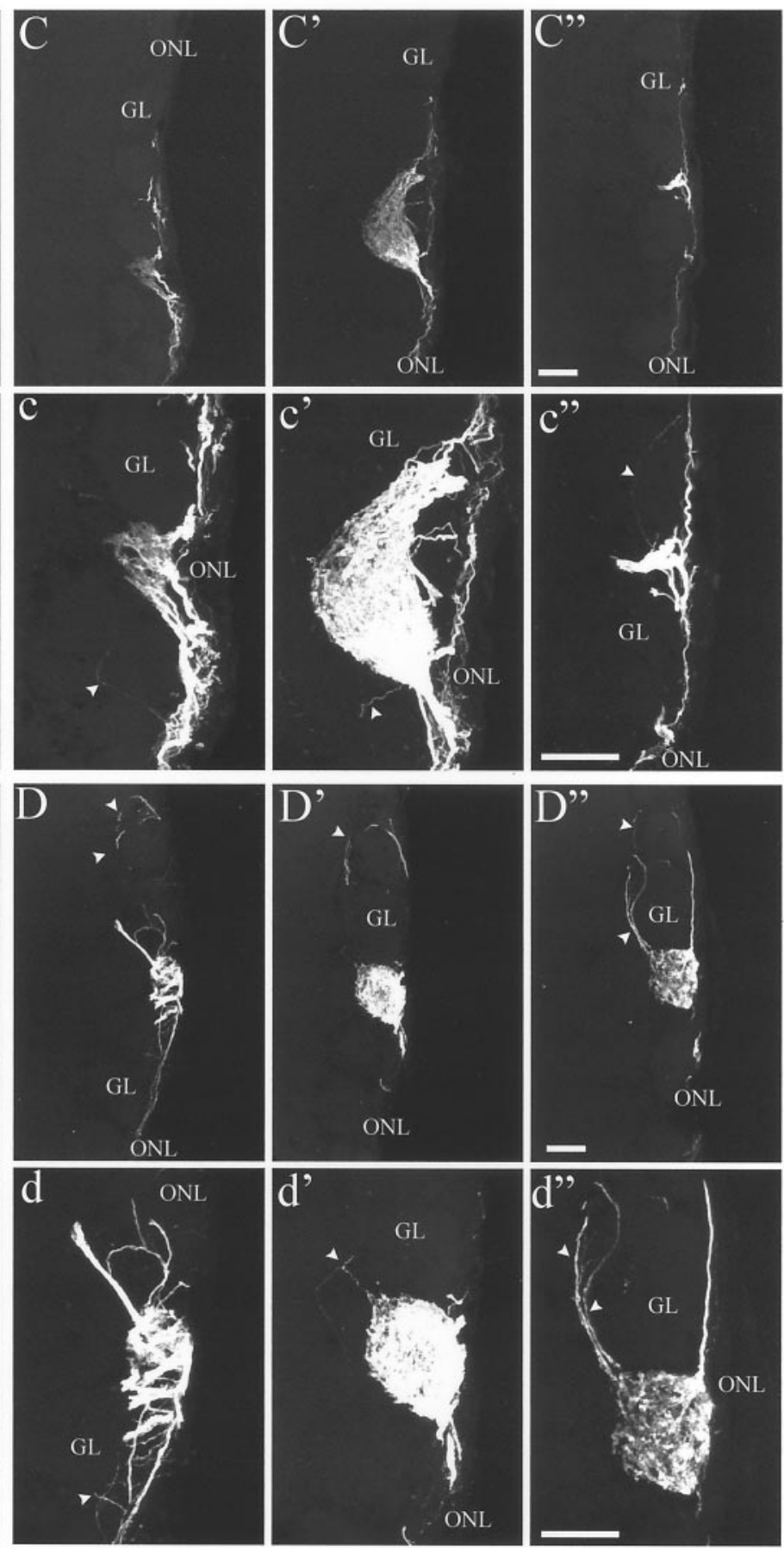

Figure 3. Projected Z-series through medial $(A, a, B, b)$ and lateral $(C, c, D, d)$ M72-IRES-tauGFP glomeruli. The projections of GFP-expressing axons were followed through three serial $50 \mu \mathrm{m}$ vibratome sections (e.g., $\left.A, A^{\prime}, A^{\prime \prime}\right)$ spanning the entire glomerular volume. We present lower-magnification $\left(A-A^{\prime \prime}, B-B^{\prime \prime}, C-C^{\prime \prime}, D-D^{\prime \prime}\right)$ images so that axons distant from the glomerular target can be viewed, as well as higher-magnification images $\left(a-a^{\prime \prime}, b-b^{\prime \prime}\right.$, $\left.c-c^{\prime \prime}, d-d^{\prime \prime}\right)$ so that single axons around glomeruli can be resolved. In all glomeruli examined, a small number of axons are observed that enter the glomerular layer from regions other than the apposed ONL (see arrowheads). These atypically projecting axons were identified in all glomeruli examined, and, although many followed tortuous paths, they all appear to target the appropriate glomerulus. Note the variation in glomerular shape and volume between the individual glomeruli. Scale bars, $50 \mu \mathrm{m}$.

the same epithelial zone) and P2-IRES-tauGFP mice (an unrelated OR that is expressed in a different epithelial zone). As can be seen in Figure 4, atypical projections were seen in both M71-IRES-tauGFP mice (Fig. $4 A-C$, arrowheads) and P2-IRES-
tauGFP mice (Fig. 4D-F, arrowheads), which suggests that these types of projection paths are common within the olfactory system. Equally important, axons from both P2-IRES-tauGFP and M71IRES-tauGFP OSNs exhibited trajectories and targeting proper- 


\section{M71-IRES-tauGFP P2-IRES-tauGFP}
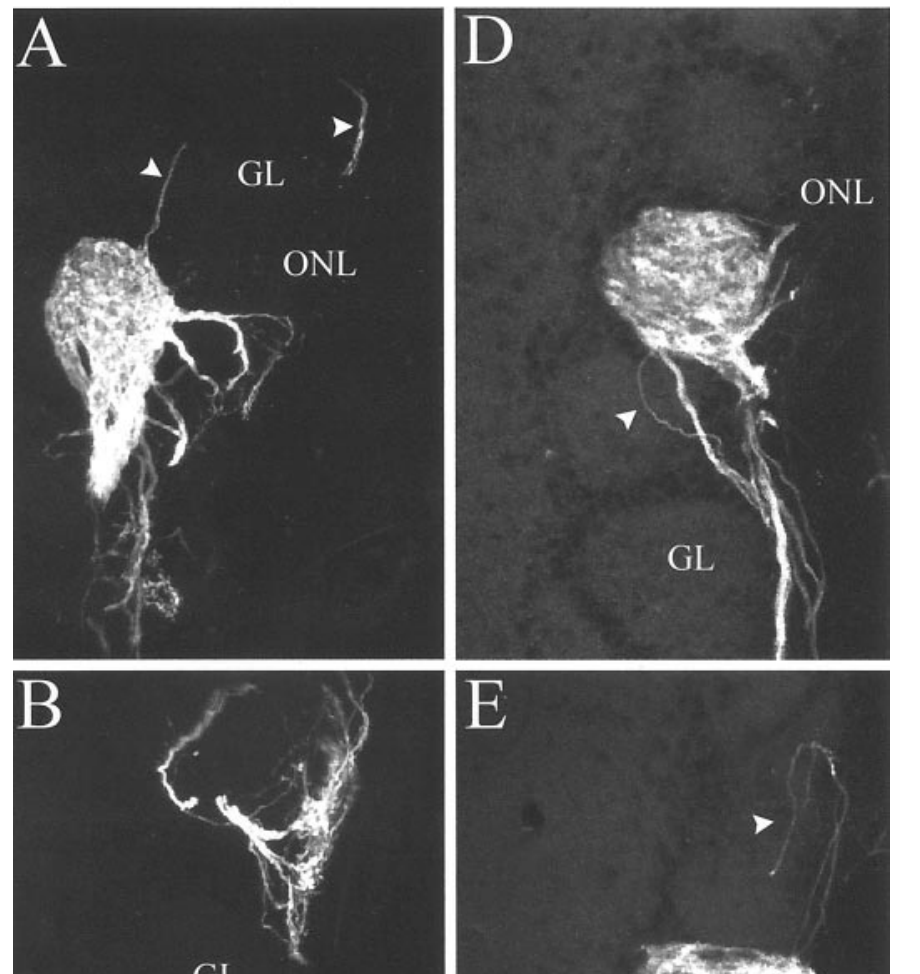

GL
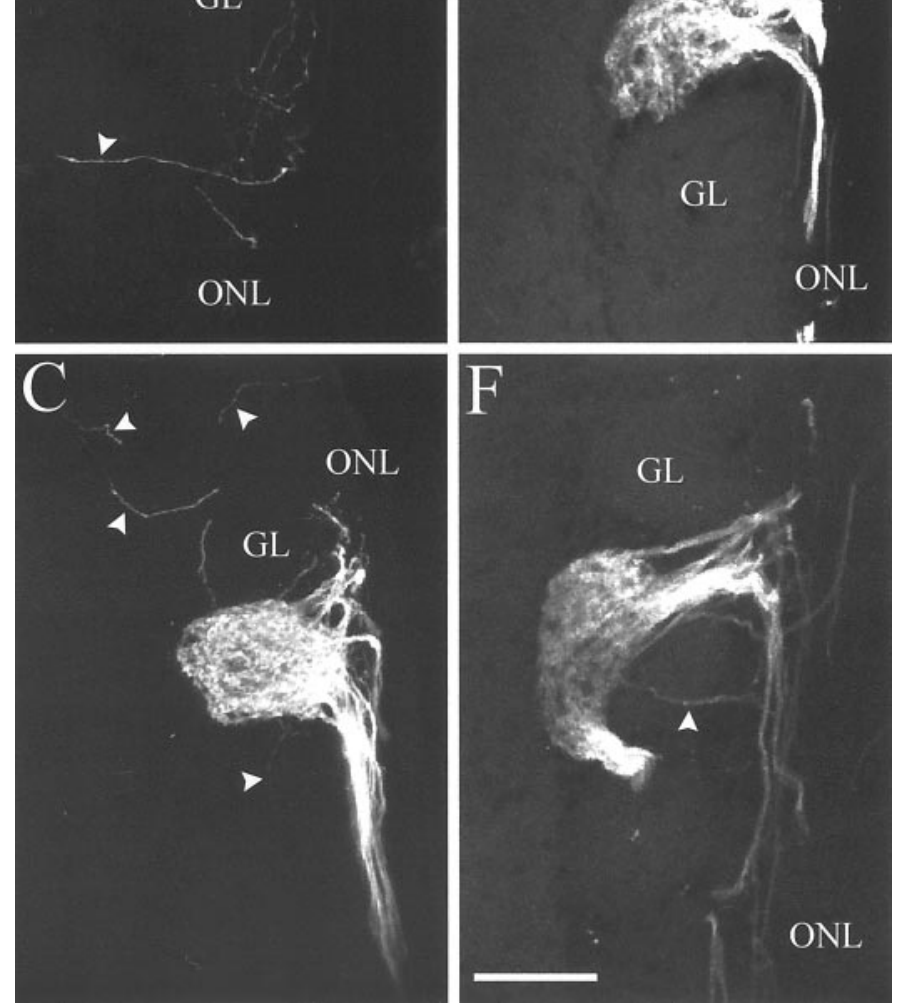

Figure 4. Projected Z-series through M71-IRES-tauGFP glomeruli $(A-C)$ and P2-IRES-tauGFP glomeruli $(D-F)$. Atypically projecting axons (see arrowheads) were also identified in mice that expressed tauGFP under the control of either the closely related M71 OR or the unrelated P2 OR. Scale bar, $50 \mu \mathrm{m}$. ties similar to those described in more detail for the M72-IREStauGFP OSN axons.

To determine whether all axons within an M72 glomerulus express GFP, we stained serial $20 \mu \mathrm{m}$ cryostat sections through M72 glomeruli with an OMP antibody and then collected images with the confocal microscope from single optical planes. We elected to stain with OMP antibodies to ensure that any GFPnegative axons observed within an M72 glomerulus would be from mature OSN neurons. In the majority of glomeruli (five of six), we saw no definitive evidence of OMP-positive-GFPnegative axons (Fig. 5). Although occasional small red axons appeared within glomeruli, this most likely represents the different compartmental localization of the respective markers within the cell. To address this issue, we examined GFP expression in glomeruli at the ultrastructural level (see below). It should be noted that many of the axons that follow anomalous paths were found to express OMP.

In one glomerulus of the six that were immunostained for OMP, there was evidence of a small region of a glomerulus that contained GFP-negative axons (Fig. 6, white arrowheads). These GFP-negative axons were surrounded by GFP-positive axons (Fig. 6B,C). To understand the nature of these axons, we examined the behavior of GFP-positive axons in glomeruli adjacent to the M72 glomerulus in tissue stained for OMP (Fig. 7). We reasoned that, if mistargeting of axons to neighboring glomeruli is a common event, we would detect evidence of GFP-labeled axons terminating in glomeruli close to the correct target. Bundles of axons were observed that clearly passed through adjacent glomeruli (Fig. 7A, $a, C, c$ ), and single axons were also observed within adjacent glomeruli (Fig. $7 B, b$ ). However, we found no evidence to suggest that these axons established terminal fields. Rather, they appeared only as axons of passage that continued on to terminate in their normal target. Thus, we conclude that the GFP-negative axons seen in Figure 6 are most likely bundles of axons passing through en route to their target glomerulus.

To assess the question of whether axons from OSNs expressing ORs other than M72 also made synapses in M72 glomeruli, we examined the ultrastructural localization of GFP within the M72 glomerulus (Fig. 8). OSN axons expressing GFP formed typical axodendritic synapses. Stained axons $(A x)$ filled with small round vesicles made typical Gray type I excitatory synapses onto unstained dendritic profiles $(D e)$. The stained axons shown in Figure $8 A-C$ are clearly distinguished from unstained axon terminals $(A x)$ from an adjacent glomerulus shown in Figure $8 D$. To determine whether the majority of axons with the M72 glomerulus express GFP, we closely examined sections for unstained axonal profiles forming synapses next to stained axonal profiles. We set these criteria because ultrastructural immunolocalization has the potential to be limited by the penetration of antibodies. Therefore, to be confident that an unstained axonal profile represented the absence of GFP (rather than incomplete antibody penetration), we limited our analyses to regions of obvious staining. In $>200$ examples of axodendritic OSN synapses, we saw only one example in which GFP labeling may have been absent from a presynaptic OSN axon terminal. As a consequence, we conclude that the overwhelming majority, if not all, of the OSN axons in M72 glomeruli are from OSNs expressing M72 ORs.

\section{DISCUSSION}

ORs expressed by subsets of OSNs are likely to have multifaceted and complex roles in the olfactory system (Mombaerts, 2001). In addition to their primary role in olfactory transduction, ORs 

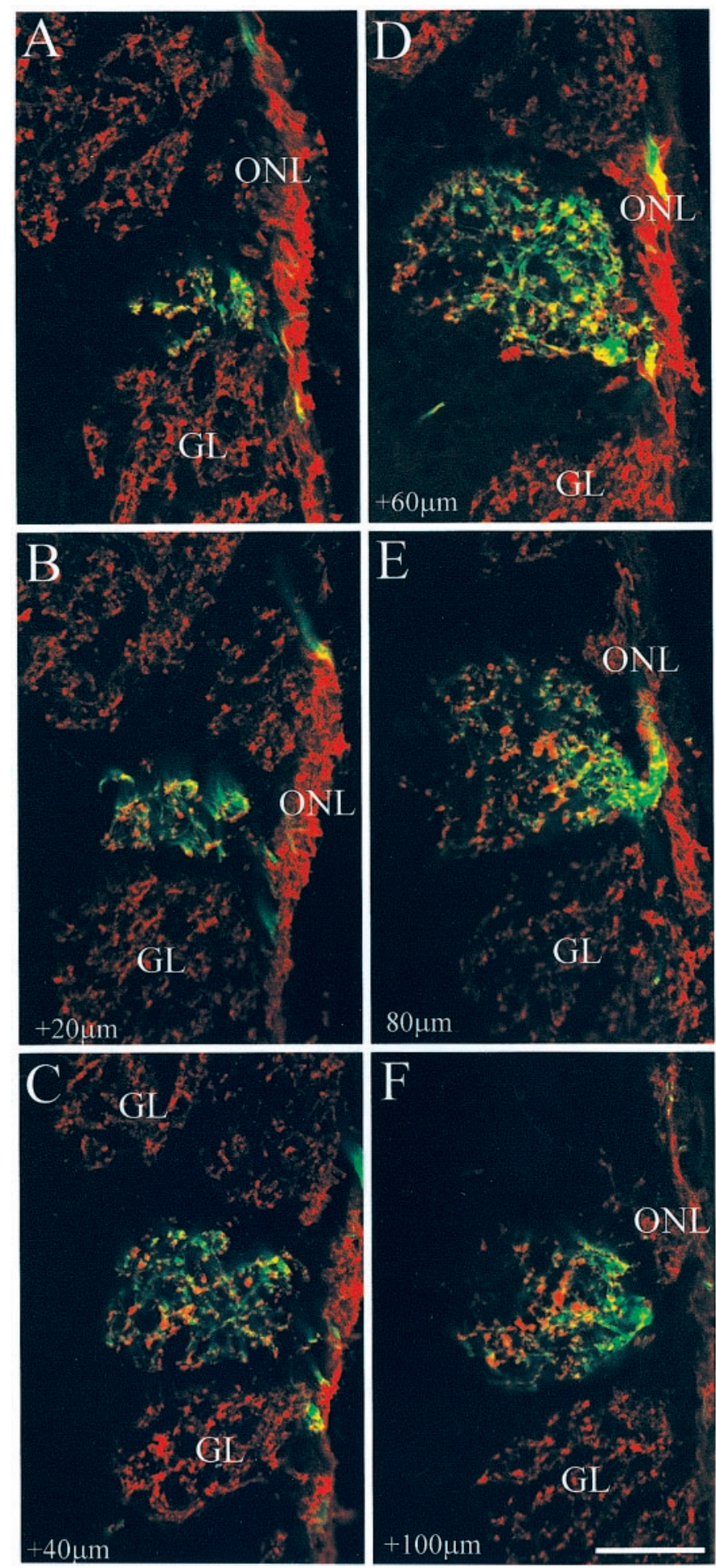

Figure 5. Serial $20 \mu \mathrm{m}$ cryostat sections $(A-F)$ through a lateral M72IRES-tauGFP glomerulus stained with an antibody to OMP (red). OSN axons expressing GFP (green) and following atypical paths also express OMP (e.g., as seen in $D$ ). Note that each of these images are single optical planes of $\sim 1 \mu \mathrm{m}$. Scale bars, $50 \mu \mathrm{m}$.

appear to have a role in axon targeting. In mice in which the coding region of one OR has been substituted with the coding region of another, OSN axons target glomeruli distinct from both the host and donor glomeruli, indicating that OR expression is one determinant, but not the only one, for final glomerular
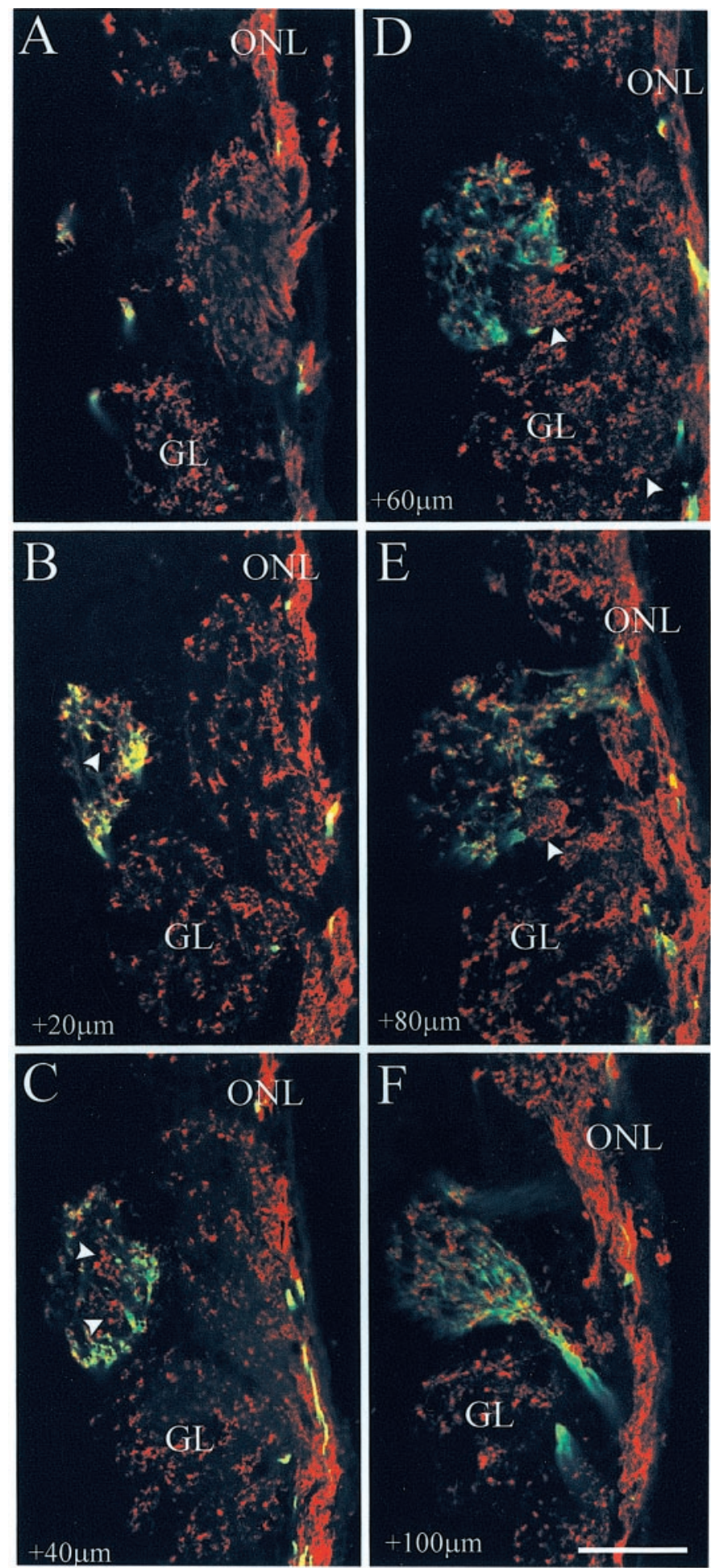

Figure 6. Serial $20 \mu \mathrm{m}$ cryostat sections through a lateral M72-IREStauGFP glomerulus stained with an antibody to OMP. Of the six glomeruli analyzed in this way ( 3 medial and 3 lateral), only this glomerulus displayed clear instances of GFP-negative axons within the M72 glomerulus (see arrowheads). These axons were clearly within the glomerulus (see arrowheads in $C$ ); however, they occupied only a small portion of the entire glomerular volume. Note that these images are single optical planes of $\sim 1 \mu \mathrm{m}$. Scale bars, $50 \mu \mathrm{m}$. 

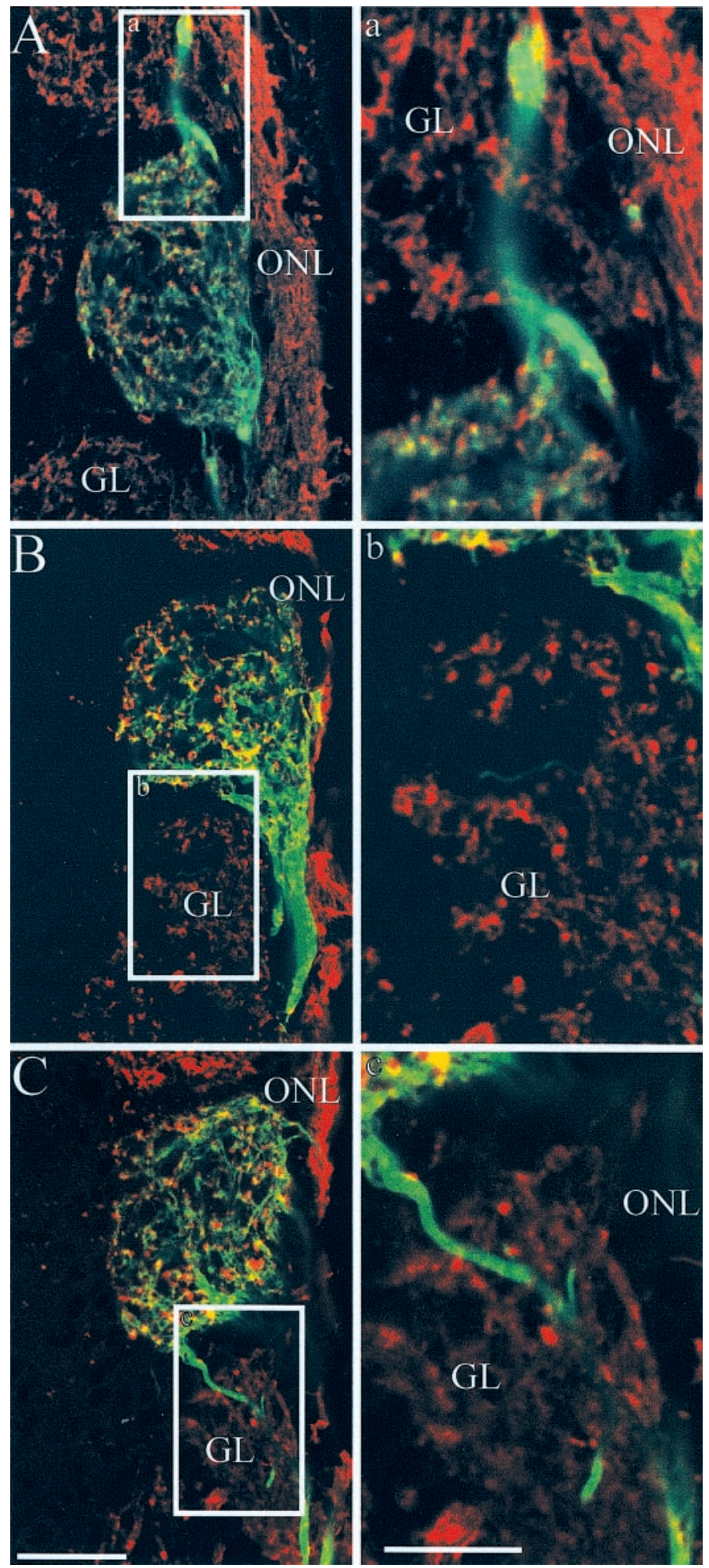

Figure 7. Examples of GFP-expressing axons projecting through glomeruli adjacent to an M72-IRES-tauGFP glomerulus. $A$, An example of a fasciculated bundle of GFP-expressing axons passing through a glomerulus en route to the target glomerulus. $B$, An example of an individual GFP-expressing axon within a glomerulus adjacent to the target glomerulus. $C$, An example of a fasciculated bundle of GFP-expressing axons with branch points within an adjacent glomerulus. $a, b$, and $c$ are enlargements of boxed regions of $A, B$, and $C$, respectively. Note that these images are single optical planes of $\sim 1 \mu \mathrm{m}$. Scale bars: $A-C, 50 \mu \mathrm{m} ; a-c, 25 \mu \mathrm{m}$.
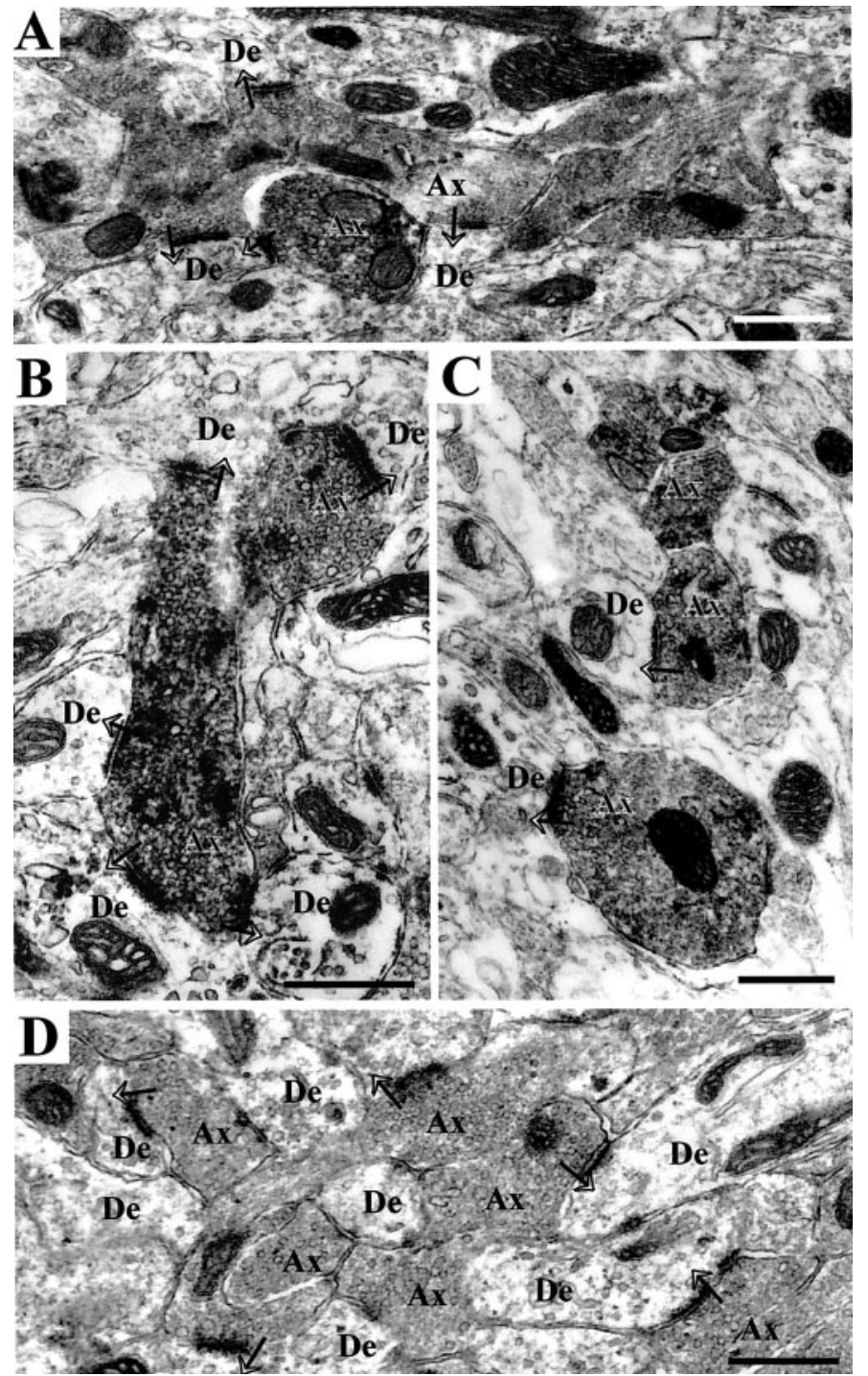

Figure 8. Synaptic profiles of GFP-expressing axons within M72 glomeruli. $A-C$, Examples of GFP-stained axons (i.e., electron dense) making Gray type 1 synapses with the electron lucent dendrites of mitral-tufted cells. The polarity of the synapses is indicted with an arrow. D, An example of unstained axons from an adjacent glomerulus. Note the difference in the electron density of the stained terminals in $A-C$ versus the unstained terminals in $D$. De, Mitral-tufted cell dendrite; $A x$, axon; $\rightarrow$, Gray type 1 synapse. Scale bars, $0.5 \mu \mathrm{m}$

targeting (Mombaerts et al., 1996; Wang et al., 1998). Thus, the specificity of the expressed OR plays a role in targeting and glomerular innervation, although the mechanisms through which OSN axon guidance are mediated remain to be fully elucidated.

\section{Homotypic fasciculation}

One possible mechanism OSN axons may use in glomerular targeting is homotypic fasciculation; axons from OSNs expressing the same OR may fasciculate en route from the olfactory epithelium to the $\mathrm{OB}$ (either by direct OR protein interactions or mediated through other adhesion molecules) and thereby innervate the same glomerulus. To test this hypothesis, we examined the distribution of OSN axons expressing GFP, focusing on whether the GFP-labeled axons fasciculated with each other (i.e., homotypic fasciculation) before terminating in a glomerulus or whether they fasciculated with axons that did not express GFP 
(i.e., heterotypic fasciculation). We undertook this study in genetargeted mice in which tauGFP is expressed in conjunction with the M72 OR. By examining intrinsic GFP fluorescence in serial sections through the $\mathrm{OB}$, trajectories of individual axons, or small fascicles of axons, could be followed. In conjunction with these confocal microscopy studies, we also localized GFP at the ultrastructural level in both the ONL and GL. Using both of these approaches, axons were found to follow diverse trajectories within the ONL. In the peripheral, or outer, ONL, the majority of axons coursed independently, showing little evidence of homotypic fasciculation. As axons approached the target glomerulus, they entered the inner ONL in which the majority of GFPpositive coalesced into larger mesaxons before coursing into the glomerulus. Potter et al. (2001), using two-photon confocal microscopy and en face views of the ONL and GL, also noted that fascicles of OSN axons followed variable pathways as they approached a target glomerulus. Our demonstration that homotypic fasciculation between axons increases as they enter the inner ONL extends these findings. At the ultrastructural level, individual GFP-expressing axons could be traced over long distances within the outer ONL in heterotypic axon fascicles in which they were closely apposed to axons that did not express GFP. Within the inner ONL, the majority of GFP-expressing axons were observed directly apposed to other GFP-expressing axons. However, not all axons within a fascicle were GFP positive, perhaps indicating that additional sorting of axons occurs still deeper within the ONL, before entry into the glomerular layer.

Of particular significance, a small proportion of axons targeted their glomeruli by following anomalous routes through the glomerular and external plexiform layers. These GFP-expressing axons were observed to pass around, between, and through neighboring glomeruli before entering the appropriate targets. Although all of these axons appeared to terminate in the appropriate glomerulus, it is possible (but unlikely) that some of these axons were exiting the glomerulus and projecting back to the ONL. To determine whether the occurrence of torturous trajectories by subpopulations of axons was unique to M72-expressing axons, we also examined GFP-expressing axons in M71-IREStauGFP and P2-IRES-tauGFP mice. Similarly, we found small populations of axons in these mice that also targeted along individual and often complicated paths. Thus, the data from all three lines of mice suggest that the trajectories that axons can follow are diverse and more idiosyncratic than recognized previously. These data further lead to the conclusion that a stable homotypic fasciculation between axons, per se, is not a prerequisite for correct axonal targeting. This does not preclude the possibility that homotypic fasciculation may facilitate targeting; it serves to underscore our consistent and reproducible observation that single axons target correctly in the absence of stable fasciculation with other GFP-expressing axons. Of additional interest will be understanding the role of OSN axon fasciculation, both homotypic and heterotypic, among populations of OSN axons during the initial formation of the glomerular map, as well as the maintenance of that map in the mature animal (Gogos et al., 2000; Schaefer et al., 2001). The absence of stable homotypic fasciculation in our images does not exclude the possibility that transient periods of fasciculation may be necessary for correct glomerular targeting. Furthermore, our observations do not rule out homotypic interactions between existing axonal tracks and navigating growth cones. These issues can be addressed in developmental studies, ideally using dynamic imaging technologies.

\section{Specificity of glomerular targeting}

Potter et al. (2001), using in situ two-photon microscopy of GFP-positive axons in adult mice, found that labeled axons targeted glomeruli with specificity, and no evidence of mistargeting to neighboring glomeruli was observed. To further characterize the specificity of OSN projections to identified glomeruli, we asked to what extent GFP-negative axons were found in the M72 glomeruli. Using OMP as a secondary marker of mature axons, our data show clearly that, in five of six glomeruli, the majority of axons within M72 glomeruli expressed GFP. Although some puncta appeared not to express both markers, we believe this reflects the different probes used in this part of the study. Only one of the six glomeruli that were stained with OMP was found to contain a large fascicle of GFP-negative-OMP-positive axons, which spanned multiple cryostat sections through the glomerulus. We cannot exclude that these axons represented a subcompartmentalized innervation of OSN axons within a glomerulus. However, given our identification of GFP-positive axons passing through neighboring glomeruli en route to their target, as well as the low frequency with which this was observed, it seems reasonable to hypothesize that these GFP-negative axons may have been passing through the M72 glomerulus en route to their target glomerulus.

To examine the specificity of glomerular targeting at higher resolution than is afforded by confocal microscopy, we undertook localization of GFP at the ultrastructural level. Although IRES constructs have been widely used to image OSN axons expressing reporter genes at the light and confocal level (Mombaerts et al., 1996; Wang et al., 1998; Royal and Key, 1999; Conzelmann et al., 2000; Serizawa et al., 2000; Strotmann et al., 2000; Zheng et al., 2000; Potter et al., 2001), to date there have been no reports of ultrastructural localization of the reporter genes in the olfactory system. At the ultrastructural level, no evidence was found of innervation of M72 glomeruli by GFPnegative axons. Because immunolocalization of GFP within glomeruli at the ultrastructural level has a different technical limitation than that seen with confocal microscopy (i.e., antibodies have limited penetration and therefore unstained axons may in fact express GFP), we restricted our analyses to regions in which stained axonal profiles were apparent, indicating adequate antibody penetration. In more than 200 synapses examined, only one example was found in which the afferent terminal ending on a mitral-tufted cell dendrite was not definitively GFP positive. This is likely to reflect the inherent limitations of ultrastructural immunocytochemistry. Therefore, we conclude from these data that the overwhelming majority of axons within the M72 glomerulus are from OSNs that express the M72 OR.

The molecular mechanisms by which OSN axons target glomeruli remain unclear. Although there is unequivocal evidence that OR proteins must be expressed for targeting to occur, the mechanism through which ORs mediate axon guidance are unknown. The data presented here argue against a simple mechanism of stable homotypic fasciculation between axons from OSNs expressing the same OR being sufficient for glomerular targeting. Axons from OR-specific OSNs take varied trajectories both within the ONL, as well as the GL, before reaching their correct glomerular target. Varied trajectories suggest that a variety of guidance mechanisms may be used for these axons to reach their targets. Consistent with this hypothesis are the plethora of guidance molecules that have been localized to the olfactory system at different stages of development. For example, members of the 
semaphorin (Pasterkamp et al., 1999), netrin (Gad et al., 1997), and ephrin (Zhang et al., 1996; St. John et al., 2000; St. John and Key, 2001) families of repulsive guidance cues and their receptors have been differentially localized in the olfactory system. Similarly, many cell adhesion molecules (for review, see Key, 1998; Mori et al., 1999), growth factors (for review, see Plendl et al., 1999), carbohydrate moieties (for review, see Plendl and Sinowatz, 1998), neurotrophins (for review, see Treloar et al., 2000), and extracellular matrix molecules (Miragall et al., 1990; Gong and Shipley, 1996; Treloar et al., 1996; Kafitz and Greer, 1997, 1998; Tisay and Key, 1999) have been differentially localized within the olfactory pathway. All of these factors may influence OSN axon growth and guidance. Given the large number of potential guidance cues, as well as the varied paths that OSN axons follow when targeting a glomerulus, it is likely that combinatorial subsets of cues and mechanisms are used by OSNs to navigate to a glomerulus, before forming a synapse.

In summary, these studies have shown that a stable homotypic fasciculation of OSN axons is not required for correct glomerular targeting to occur. The tortuous trajectories followed by single axons emphasize the heterogeneous mechanisms that are available for directing axon targeting. In the context of assessing the behavior of identified axons as they approach their target glomerulus, we recognized subdivisions in the ONL; axons traveled alone or in small fascicles in the outer ONL, whereas fasciculation among homotypic axons occurred extensively in the inner ONL. Finally, these data are the first to show that labeled OSN axons in OR gene-tagged mice make synapses in the glomeruli and that the axonal composition of the glomerulus is extremely homogeneous in terms of OR expression.

\section{REFERENCES}

Berod A, Hartman BK, Pujol JF (1981) Importance of fixation in immunohistochemistry: use of formaldehyde solutions at variable $\mathrm{pH}$ for the localization of tyrosine hydroxylase. J Histochem Cytochem 29:844-850.

Conzelmann S, Levai O, Bode B, Eisel U, Raming K, Breer H, Strotmann $\mathrm{J}$ (2000) A novel brain receptor is expressed in a distinct population of olfactory sensory neurons. Eur J Neurosci 12:3926-3934.

Firestein S (2001) How the olfactory system makes sense of scents. Nature 413:211-218.

Gad JM, Keeling SL, Wilks AF, Tan SS, Cooper HM (1997) The expression patterns of guidance receptors, DCC and Neogenin, are spatially and temporally distinct throughout mouse embryogenesis. Dev Biol 192:258-273.

Gogos JA, Osbourne J, Nemes A, Mendelsohn M, Axel R (2000) Genetic ablation and restoration of the olfactory topographic map. Cell 103:609-620.

Gong Q, Shipley MT (1996) Expression of extracellular matrix molecules and cell surface molecules in the olfactory nerve pathway during early development. J Comp Neurol 366:1-14.

Guthrie KM, Anderson AJ, Leon M, Gall C (1993) Odor-induced increases in c-fos mRNA expression reveal an anatomical "unit" for odor processing in olfactory bulb. Proc Natl Acad Sci USA 90:3329-3333.

Kafitz KW, Greer CA (1997) Role of laminin in axonal extension from olfactory receptor cells. J Neurobiol 32:298-310.

Kafitz KW, Greer CA (1998) Differential expression of extracellular matrix and cell adhesion molecules in the olfactory nerve and glomerular layers of adult rats. J Neurobiol [Erratum (1998) 35:118] 34:271-282.

Keller A, Margolis FL (1975) Immunological studies of the rat olfactory marker protein. J Neurochem 24:1101-1106.

Key B (1998) Molecular development of the olfactory nerve pathway. Ann NY Acad Sci 855:76-82.

Leveteau J, MacLeod P (1966) Olfactory discrimination in the rabbit olfactory glomerulus. Science 153:175-176.

Miragall F, Kadmon G, Faissner A, Antonicek H, Schachner M (1990) Retention of $\mathrm{J} 1 /$ tenascin and the polysialylated form of the neural cell adhesion molecule (N-CAM) in the adult olfactory bulb. J Neurocytol 19:899-914.

Mombaerts P (1999) Seven-transmembrane proteins as odorant and chemosensory receptors. Science 286:707-711.

Mombaerts P (2001) How smell develops. Nat Neurosci 4:1192-1198.

Mombaerts P, Wang F, Dulac C, Chao SK, Nemes A, Mendelsohn M, Edmondson J, Axel R (1996) Visualizing an olfactory sensory map. Cell 87:675-686.

Montague AA, Greer CA (1999) Differential distribution of ionotropic glutamate receptor subunits in the rat olfactory bulb. J Comp Neurol 405:233-246.

Mori K, Nagao H, Yoshihara Y (1999) The olfactory bulb: coding and processing of odor molecule. Science 286:711-715.

Pasterkamp RJ, Ruitenberg MJ, Verhaagen J (1999) Semaphorins and their receptors in olfactory axon guidance. Cell Mol Biol 45:763-779.

Plendl J, Sinowatz F (1998) Glycobiology of the olfactory system. Acta Anat 161:234-253

Plendl J, Stierstorfer B, Sinowatz F (1999) Growth factors and their receptors in the olfactory system. Anat Histol Embryol 28:73-79.

Potter S, Zheng C, Koos D, Feinstein P, Fraser S, Mombaerts P (2001) Structure and emergence of specific olfactory glomeruli in the mouse. J Neurosci 21:9713-9723.

Pyrski M, Xu Z, Walters E, Gilbert DJ, Jenkins NA, Copeland NG, Margolis FL (2001) The OMP-lacZ transgene mimics the unusual expression pattern of OR-Z6, a new odorant receptor gene on mouse chromosome 6: implication for locus-dependent gene expression. J Neurosci 21:4637-4648.

Ressler KJ, Sullivan SL, Buck LB (1994) Information coding in the olfactory system: evidence for a stereotyped and highly organized epitope map in the olfactory bulb. Cell 79:1245-1255.

Royal SJ, Key B (1999) Development of P2 olfactory glomeruli in P2internal ribosome entry site-tau-LacZ transgenic mice. J Neurosci 19:9856-9864.

Rubin BD, Katz LC (1999) Optical imaging of odorant representation in the mammalian olfactory bulb. Neuron 23:499-511.

Schaefer ML, Finger TE, Restrepo D (2001) Variability of position of the P2 glomerulus within a map of the mouse olfactory bulb. J Comp Neurol 436:351-362.

Serizawa S, Ishii T, Nakatani H, Tsuboi A, Nagawa F, Asano M, Sudo K, Sakagami J, Sakano H, Ijiri T, Matsuda Y, Suzuki M, Yamamori T, Iwakura Y, Sakano H (2000) Mutually exclusive expression of odorant receptor transgenes. Nat Neurosci 3:687-693.

Shepherd GM (1981) The olfactory glomerulus: its significance for sensory processing. In: Brain mechanisms of sensation (Katsuki Y, Norgren S, eds), pp 209-223. New York: Wiley.

St. John JA, Key B (2001) EphB2 and two of its ligands have dynamic protein expression patterns in the developing olfactory system. Dev Brain Res 126:43-56.

St. John JA, Tisay KT, Caras IW, Key B (2000) Expression of EphA5 during development of the olfactory nerve pathway in rat. J Comp Neurol 416:540-550.

Strotmann J, Conzelmann S, Beck A, Feinstein P, Breer H, Mombaerts P (2000) Local permutations in the glomerular array of the mouse olfactory bulb. J Neurosci 20:6927-6938.

Tisay KT, Key B (1999) The extracellular matrix modulates olfactory neurite outgrowth on ensheathing cells. J Neurosci 19:9890-9899.

Treloar HB, Nurcombe V, Key B (1996) Expression of extracellular matrix molecules in the embryonic rat olfactory pathway. J Neurobiol 31:41-55.

Treloar HB, Bartolomei JC, Lipscomb BW, Greer CA (2001) Mechanisms of axonal plasticity: Lessons from the olfactory pathway. The Neuroscientist 7:55-63.

Uchida N, Takahashi YK, Tanifuji M, Mori K (2000) Odor maps in the mammalian olfactory bulb: domain organization and odorant structural features. Nat Neurosci 3:1035-1043.

Vassar R, Chao SK, Sitcheran R, Nuñez JM, Vosshall LB, Axel R (1994) Topographic organization of sensory projections to the olfactory bulb. Cell 79:981-991.

Wang F, Nemes A, Mendelsohn M, Axel R (1998) Odorant receptors govern the formation of a precise topographic map. Cell 93:47-60.

Zhang JH, Cerretti DP, Yu T, Flanagan JG, Zhou R (1996) Detection of ligands in regions anatomically connected to neurons expressing the Eph receptor Bsk: potential roles in neuron-target interaction. J Neurosci 16:7182-7192.

Zhao H, Reed RR (2001) X inactivation of the OCNC1 channel gene reveals a role for activity-dependent competition in the olfactory system. Cell 104:651-660.

Zheng C, Feinstein P, Bozza T, Rodriguez I, Mombaerts P (2000) Peripheral olfactory projections are differentially affected in mice deficient in a cyclic-nucleotide gated channel subunit. Neuron 26:81-91. 